\title{
Anharmonic phonon effects on linear thermal expansion of trigonal bismuth selenide and antimony telluride crystals
}

\author{
Chee Kwan Gan ${ }^{1, \text { a) }}$ and Ching Hua Lee ${ }^{1}$ \\ Institute of High Performance Computing, 1 Fusionopolis Way, \#16-16 Connexis, \\ Singapore 138632
}

(Dated: 15 March 2018 (b: CMS version))

We adopted and extended an efficient Grüneisen formalism to study the phonon anharmonicity and linear thermal expansion coefficients (TECs) of trigonal bismuth selenide $\left(\mathrm{Bi}_{2} \mathrm{Se}_{3}\right)$ and antimony telluride $\left(\mathrm{Sb}_{2} \mathrm{Te}_{3}\right)$. Anharmonicity of the systems is studied via extensive calculation of Grüneisen parameters that exploit symmetry-preserving deformations. Consistent with experimental findings, a large anisotropy between the TECs in the $a$ and $c$ directions is found. The larger anharmonicity inherent in $\mathrm{Sb}_{2} \mathrm{Te}_{3}$, as compared to $\mathrm{Bi}_{2} \mathrm{Se}_{3}$ is offset by the volumetric effect, resulting in comparable temperature dependence of their linear TECs. The Debye temperatures deduced from our first-principles data also agree very well with the existing tabulated values. The highly efficient methodology developed in this work, applied for the first time to study the linear TECs of two trigonal thermoelectric systems, opens up exciting opportunities to address the anharmonic effects in other thermoelectrics and other low-symmetry materials.

PACS numbers: 63.20.D-, 65.40.-b, 65.40.De

Keywords: Bismuth selenide, antimony telluride, phonon calculations, thermal expansion, topological insulators, thermoelectric materials, Grüneisen parameter, Debye temperature

\section{INTRODUCTION}

Bismuth selenide $\left(\mathrm{Bi}_{2} \mathrm{Se}_{3}\right)$ and antimony telluride $\left(\mathrm{Sb}_{2} \mathrm{Te}_{3}\right)$ belong to a large family of metal dichalcogenides that hosts excellent thermoelectric materials 1 and topological insulators ${ }^{2}$. As paradigmatic examples of materials that simultaneously host enigmatic 3D $Z_{2}$ topological states, these two materials have been extensively studied experimentally $\sqrt{5-8}$ and theoretically $\sqrt{9 \mid 10}$ due to their technological importance and fundamental interest. The linear and volumetric thermal expansion coefficients (TECs) of $\mathrm{Bi}_{2} \mathrm{Se}_{3}$ and $\mathrm{Sb}_{2} \mathrm{Te}_{3}$ have been determined experimentally ${ }^{\sqrt{5}}$ where a high anisotropy is found between linear TECs in the $a$ and $c$ directions for these two systems.

For engineering applications of these materials, good device performance hinges on a solid understanding of thermal expansion behavior because phonon dynamics is intimately affected by temperature-induced crystal deformations. As found in $\frac{11 \mid 12}{1}$, knowledge of the linear thermal expansion and phonon anharmonicity can be captured through phonon frequency lineshifts through the Grüneisen parameters. Such calculations of the thermal expansion properties are commonly performed using a quasi-harmonic approximation (QHA), which involves many phonon calculations on many possible combinations of lattice parameters. But due to its complexity, the QHA is efficient only when dealing with highly symmetric systems such as cubic lattice structures. However, many technological important crystals are not cubic, and other more efficient approaches are necessary. In this pa-

\footnotetext{
a) Electronic mail: ganck@ihpc.a-star.edu.sg
}

per, we adopted and extended an efficient Grüneisen approach by 13 to study $\mathrm{Bi}_{2} \mathrm{Se}_{3}$ and $\mathrm{Sb}_{2} \mathrm{Te}_{3}$ with a minimal set of relatively expensive (compared to standard density-functional total-energy calculations) phonon calculations. Through it, we were able to perform a systematic investigation on the anharmonicity of these two materials with relatively low symmetry, and make consistent comparisons between some of their important thermal properties such as linear TECs.

\section{METHODOLOGY}

The trigonal $\mathrm{Bi}_{2} \mathrm{Se}_{3}$ and $\mathrm{Sb}_{2} \mathrm{Te}_{3}$ belong to the symmorphic space group $R \overline{3} m$ (No. 166). There are three inequivalent atoms: an $\mathrm{Sb}$ atom occupies $6 c(0,0, \mu)$ site, a Te atom occupies $3 a(0,0,0)$ site, and a second $\mathrm{Te}$ atom occupies $6 c(0,0, \nu)$ site. This gives a total of 15 atoms in the conventional hexagonal unit cell. However, in order to reduce the amount of computing time, we use a primitive rhombohedral cell of five atoms that is three times smaller than the conventional hexagonal cell. The rhombohedral cell length $a_{r}$ and angle $\alpha_{r}$ can be deduced from the hexagonal lattice parameters $a_{h}$ and $c_{h}$, and vice versa. The relations are: $a_{h}=2 a_{r} \sin \left(\alpha_{r} / 2\right), c_{h}=a_{r} \sqrt{3+6 \cos \alpha_{r}}$. On the other hand, $a_{r}=\left(a_{h} / 3\right) \sqrt{\eta^{2}+3}, \cos \alpha_{r}=\left(2 \eta^{2}-3\right) /\left(2 \eta^{2}+6\right)$ where $\eta=c_{h} / a_{h}$.

We perform density-functional theory (DFT) calculations within the local density approximation as implemented in the plane-wave basis suite QUANTUM ESPRESSO ${ }^{17}(\mathrm{QE})$, with wavefunction and density cutoffs of 60 and 480 Rydberg, respectively. A $10 \times 10 \times 10$ Monkhorst-Pack mesh is used for the $k$-point sampling. The pseudopotentials for $\mathrm{Bi}, \mathrm{Se}, \mathrm{Sb}$, and Te are gen- 
erated using the pslibrary.1.0.0 that is based on the Rappe-Rabe-Kaxiras-Joannopoulos $\frac{18}{18}$ scheme. We relax the structures fully before carrying out the phonon calculations. For $\mathrm{Bi}_{2} \mathrm{Se}_{3}$, we obtain $(a, c)=(4.110,27.900) \AA$. This is in good agreement with the experimental19 result of $(4.143,28.636) \AA$. For $\mathrm{Sb}_{2} \mathrm{Te}_{3}$, we obtain $(a, c)=$ $(4.244,29.399) \AA$, which is in good agreement with the experimenta $\sqrt{5}$ result of $(4.242,30.191) \AA$.

According to the Grüneisen approach 13 16120121, the linear TECs in the $a$ and $c$ directions, denoted as $\alpha_{a}(T)$ and $\alpha_{c}(T)$, respectively, are given by

$$
\left(\begin{array}{c}
\alpha_{a} \\
\alpha_{c}
\end{array}\right)=\frac{1}{\Omega D}\left(\begin{array}{cc}
C_{33} & -C_{13} \\
-2 C_{13} & {\left[C_{11}+C_{12}\right.}
\end{array}\right)\left(\begin{array}{l}
I_{1} \\
I_{3}
\end{array}\right)
$$

where $D=\left(C_{11}+C_{12}\right) C_{33}-2 C_{13}^{2}$. For clarity, the ex- plicit dependence of $\alpha$ 's and $I_{i}$ 's on temperature $T$ is suppressed in Eq. 1. We will discuss more about $I_{i}$ later. The $C_{i j}$ are the elastic constants. The linear TECs are inversely proportional to the volume $\Omega$ of primitive cell at equilibrium. We note that $\mathrm{Bi}_{2} \mathrm{Se}_{3}$ has a smaller $\Omega$ than $\mathrm{Sb}_{2} \mathrm{Te}_{3}$ (i.e., $136.05 \AA^{3}$ vs $152.87 \AA^{3}$ ). From a series of symmetry-preserving deformations with strain parameters ranging from -0.01 to 0.01 , the elastic constants are deduced from parabolic fits to the energy-strain 22 curves. For $\mathrm{Bi}_{2} \mathrm{Se}_{3}, C_{11}+C_{12}=121.74, C_{13}=30.18$, and $C_{33}=54.45 \mathrm{GPa}$. For $\mathrm{Sb}_{2} \mathrm{Te}_{3}, C_{11}+C_{12}=110.73$, $C_{13}=32.16$, and $C_{33}=60.97 \mathrm{GPa}$. We note that the expression for TECs in Eq. 1 is identical to the hexagonal case $^{16}$ since a trigonal cell can be perfectly embedded in a hexagonal cell.
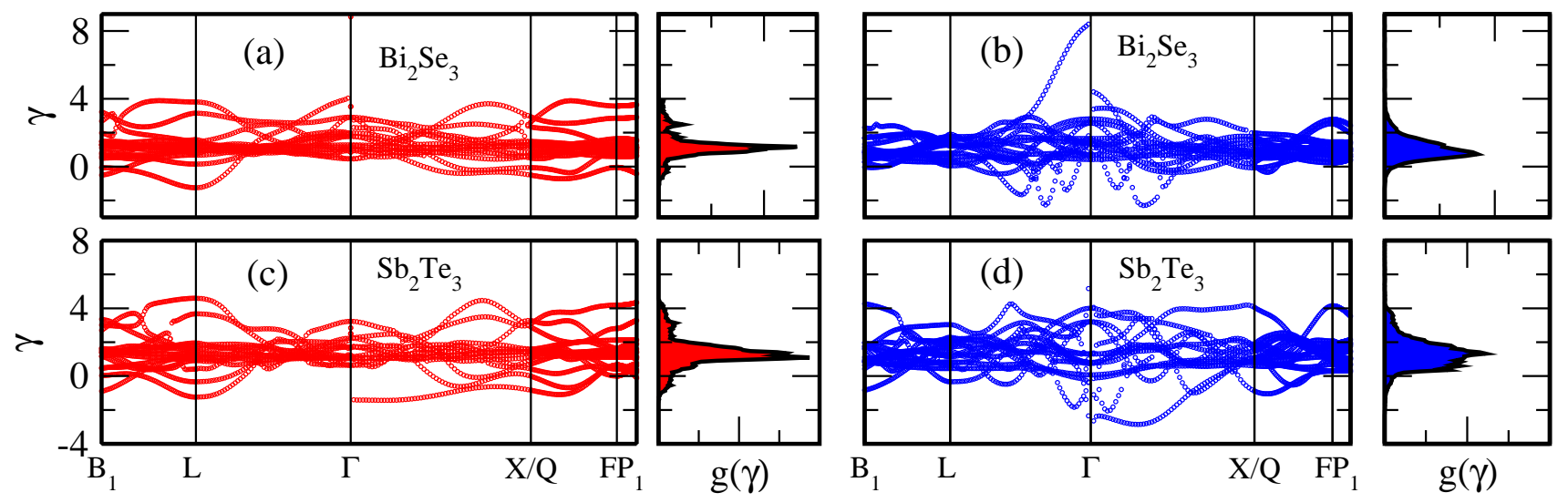

FIG. 1. The Grüneisen parameters for $\mathrm{Bi}_{2} \mathrm{Se}_{3}$ due to (a) an $x y$ biaxial strain and (b) a $z$ uniaxial strain. The corresponding results for $\mathrm{Sb}_{2} \mathrm{Te}_{3}$ are shown in (c) and (d), respectively. The label and coordinates of the $k$ points are taken from Ref. 23. The densities of Grüneisen parameters, $g(\gamma)$, shown on the right side of each figure are obtained with a sampling of $30 \times 30 \times 30$ $k$ points.

Central to our Grüneisen formalism is the temperature dependent heat capacity weighted by the Grüneisen parameter,

$$
I_{i}(T)=\frac{\Omega}{(2 \pi)^{3}} \sum_{\lambda} \int_{\mathrm{BZ}} d \boldsymbol{k} \gamma_{i, \lambda \boldsymbol{k}} c\left(\nu_{\lambda \boldsymbol{k}}, T\right)
$$

where the integral is over the first Brillouin zone (BZ). Here $\gamma_{i, \lambda \boldsymbol{k}}=-n^{-1} \nu_{\lambda \boldsymbol{k}}^{-1} \partial \nu_{\lambda \boldsymbol{k}} / \partial \epsilon_{i}$ are the modedependent and deformation-dependent Grüneisen parameters, which measures the rate of change of the phonon frequency $\nu_{\lambda k}$ (of mode index $\lambda$ and wavevector $\boldsymbol{k}$ ) with respect to the strain parameter $\epsilon_{i} . n$ equals to 1 (2) for a uniaxial (biaxial) strain. The specific heat contributed by a phonon mode of frequency $\nu$ is $c(\nu, T)=k_{B}(r / \sinh r)^{2}, r=h \nu / 2 k_{B} T . k_{B}$ and $h$ are the Boltzmann and Planck constants, respectively. To keep track of the origin of anharmonicity more precisely, we further define the the density of phonon states weighted by Grüneisen parameter, $\Gamma_{i}(\nu)$, given by

$$
\Gamma_{i}(\nu)=\frac{\Omega}{(2 \pi)^{3}} \sum_{\lambda} \int_{\mathrm{BZ}} d \boldsymbol{k} \delta\left(\nu-\nu_{\lambda \boldsymbol{k}}\right) \gamma_{i, \lambda \boldsymbol{k}}
$$

such that $I_{i}(T)=\int_{\nu_{\min }}^{\nu_{\max }} d \nu \Gamma_{i}(\nu) c(\nu, T) . \quad \nu_{\min }\left(\nu_{\max }\right)$ is the minimum (maximum) frequency in the phonon spectrum. The functions $\Gamma_{i}(\nu)$ provide a deeper understanding about $I_{i}(T)$ since it isolates the anharmonicitydependent contributions from the harmonic specific heat capacity $c(\nu, T)$, which has a well-known universal form $\frac{15}{15}$. Finally we note that $I_{i}(T)$ is related to the macroscopic ${ }^{24}$ Grüneisen parameters, $\gamma_{m, i}(T)$ by the relation $\gamma_{m, i}(T)=I_{i}(T) / C_{v}(T)$ where $C_{v}(T)=$ $\frac{\Omega}{(2 \pi)^{3}} \sum_{\lambda} \int_{\mathrm{BZ}} d \boldsymbol{k} c\left(\nu_{\lambda \boldsymbol{k}}, T\right)$ is the specific heat at constant volume. Therefore $\gamma_{m, i}(T)$ can be interpreted as an average over Grüneisen parameters weighted by the mode 
dependent heat capacity. Its physical meaning is clearest in the large- $T$ limit, where $\gamma_{m, i}$ reduces to a simple arithmetic average of all Grüneisen parameters in the BZ since the heat capacities for each mode approaches unity (in units of $k_{B}$ ) in this limit.

To calculate the Grüneisen parameters resulted from a deformation of the crystal ${ }^{21}$ due to an $x y$ biaxial strain, a strain-parameter set of $\left(\epsilon_{1}, \epsilon_{1}, 0,0,0,0\right)$ (in Voigt's notation) is used, where the rhombohedral cell has a new lattice parameters $a_{r}^{\prime}=a_{r} \sqrt{\left[\eta^{2}+3\left(1+\epsilon_{1}\right)^{2}\right] /\left(\eta^{2}+3\right)}$ and $\cos \alpha_{r}^{\prime}=\left[2 \eta^{2}-3\left(1+\epsilon_{1}\right)^{2}\right] /\left[2 \eta^{2}+6\left(1+\epsilon_{1}\right)^{2}\right]$. For a $z$ uniaxial strain, we use the strain-parameter set of $\left(0,0, \epsilon_{3}, 0,0,0\right)$, where the rhombohedral cell has $a_{r}^{\prime}=$ $a_{r} \sqrt{\left[\eta^{2}\left(1+\epsilon_{3}\right)^{2}+3\right] /\left(\eta^{2}+3\right)}$ and $\cos \alpha_{r}^{\prime}=\left[2 \eta^{2}(1+\right.$ $\left.\left.\epsilon_{3}\right)^{2}-3\right] /\left[2 \eta^{2}\left(1+\epsilon_{3}\right)^{2}+6\right]$. Importantly, these two deformations preserve the space group of the crystal so that we can use the QE symmetry switch of IBRAV $=5$. We use small strains of $e_{1}= \pm 0.25 \%$ and $e_{3}= \pm 0.5 \%$ for the calculation Grüneisen parameters using finite-differences. For phonon calculations under the QE implementation, we use a $q$ mesh of $5 \times 5 \times 5$, which is equivalent to a $5 \times 5 \times 5$ supercell $\frac{25}{2}$ for the determination of interatomic force constants.

\section{RESULTS}

The Grüneisen parameters along the representative high-symmetry directions for $\mathrm{Bi}_{2} \mathrm{Se}_{3}$ and $\mathrm{Sb}_{2} \mathrm{Te}_{3}$ due to an $x y$ biaxial strain are shown in Fig. 11(a) and (c), respectively. Similarly, the results due to a $z$ uniaxial strain are shown in Fig. 1(b) and (d), respectively. The densities of Grüneisen parameters (displayed on the right side of each subfigure) show that most Grüneisen parameters range between 0 to 4 , with a dominant peak centered around 1. There is a small population of negative Grüneisen parameters, which may lead to negative linear TECs $\$$.

For the above discussion, the temperature dependence of the TECs hinges on the integrated quantities $I_{i}(T)$, which could be calculated from a direct summation over BZ or through an integration over frequency $\nu$ of the product of heat capacity $c(\nu, T)$ and the density of phonon states weighted by Grüneisen parameter, $\Gamma_{i}(\nu)$. We find the second approach is more illuminating. The density of phonon states for $\mathrm{Bi}_{2} \mathrm{Se}_{3}$ in Fig. 2(a) shows there is a phonon gap of 89 to $94 \mathrm{~cm}^{-1}$ which is due mainly to a large mass difference between $\mathrm{Bi}$ (atomic mass of 208.98) and Se (atomic mass of 78.97). Such phonon gap is not seen for $\mathrm{Sb}_{2} \mathrm{Te}_{3}$ [shown in 2(c)] since $\mathrm{Sb}$ (atomic mass of 121.76) and Te (atomic mass of 127.60) are consecutive elements in the periodic table. $\Gamma_{i}(\nu)$ for $\mathrm{Bi}_{2} \mathrm{Se}_{3}$ are shown in Fig. 2(b) for both $x y$ biaxial and $z$ uniaxial strains, where large Grüneisen parameters are associated with frequencies of about $100 \mathrm{~cm}^{-1}$. For $\mathrm{Sb}_{2} \mathrm{Te}_{3}$, large Grüneisen parameters are associated with frequencies of about $90 \mathrm{~cm}^{-1} . \Gamma_{i}(\nu)$ shown in Fig. 2(b) and (d) also indicate that effect of negative Grüneisen parameters are negligible for all frequencies except for
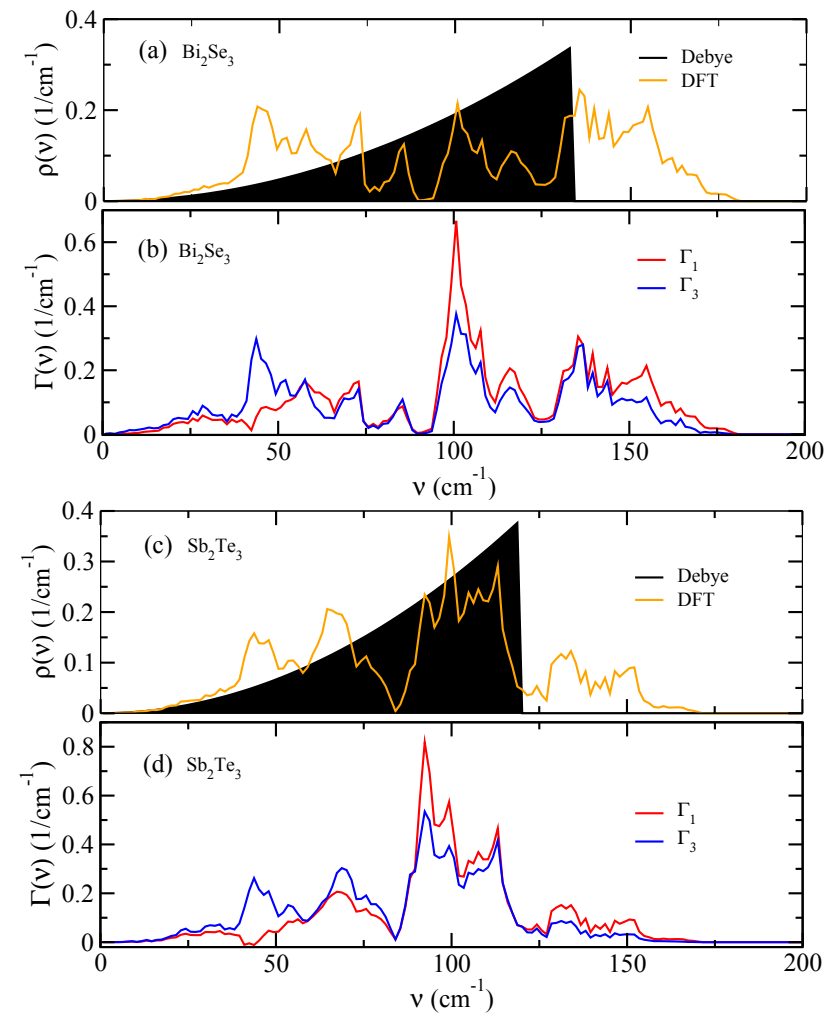

FIG. 2. (a) The phonon density of states (from DFT and Debye approximation), $\rho(\nu)$. (b) The phonon density of states weighted by Grüneisen parameter, $\Gamma_{i}(\nu)$, due to an $x y$ biaxial strain and a $z$ uniaxial strain for the $\mathrm{Bi}_{2} \mathrm{Se}_{3}$. The corresponding results are shown in (c) and (d) for $\mathrm{Sb}_{2} \mathrm{Te}_{3}$.

the $x y$ biaxial strain of $\mathrm{Sb}_{2} \mathrm{Te}_{3}$ at about $38 \mathrm{~cm}^{-1}$.

Since the temperature dependence of TECs is intricately related to that of the heat capacity at constant volume, which is typically characterized by the Debye temperature, here we suggest a simple approach to extract the effective Debye temperature. From phonon calculations based on density-functional perturbation theory (DFPT), we could obtain very accurate phonon density of states and hence heat capacity as a function of temperature [see Fig. 3(a) and (d)]. We propose to fit the DFT heat capacity data with that obtained from a Debye model approximation by minimizing the absolute error as a function of a cutoff frequency $\nu_{c}$,

$$
d\left(\nu_{c}\right)=\frac{1}{\left(3 N k_{B}\right)^{2}} \int_{0}^{\infty} d T\left[C_{v}^{D}\left(\nu_{c}, T\right)-C_{v}^{D F T}(T)\right]^{2}
$$

where the integrand is the square of the difference between of the heat capacities from DFT and from the Debye model. $N=5$ is the number of atoms in the primitive cell in current systems. According to this scheme, the Debye temperature will be naturally defined as $\theta_{D}=h \nu_{c} / k_{B}$. The heat capacity evaluated according to the Debye model is $C_{v}^{D}\left(\nu_{c}, T\right)=\int_{0}^{\nu_{c}} \rho_{D}(\nu) c(\nu, T) d \nu$ and the density of phonon states under the Debye approximation is $\rho_{D}(\nu)=A \nu^{2}$ for $0 \leq \nu \leq \nu_{c}$ and zero 
otherwise $\left(A=9 N / \nu_{c}^{3}\right)$. We note that a similar scheme for finding the Debye temperature as a function of temperature has been proposed in Ref. 26. The best cutoff frequencies are 133 and $119 \mathrm{~cm}^{-1}$ for $\mathrm{Bi}_{2} \mathrm{Se}_{3}$ and $\mathrm{Sb}_{2} \mathrm{Te}_{3}$, respectively. This translates to $\theta_{D}$ of $191 \mathrm{~K}$ and $172 \mathrm{~K}$, respectively. These values are in the correct order and agree well with the literature ${ }^{27}$ values of 182 and $160 \mathrm{~K}$, respectively. It is interesting to see that even though the phonon densities of states from the Debye approximation and DFT differ significantly [see Fig. 22(a) and (c)], the heat capacities between DFT and Debye approximation agree remarkably well with each other [see Fig. 3(a) and (d)], which demonstrates the robustness of Debye model to describe the heat capacity.

With the $\Gamma_{i}(\nu)$ data, we calculate the integrated quantities $I_{i}(T)$ as shown in Fig. 3(b) and (e) (solid lines), for $\mathrm{Bi}_{2} \mathrm{Se}_{3}$ and $\mathrm{Sb}_{2} \mathrm{Te}_{3}$, respectively. These are positive functions, which eliminate the occurrence of negative linear TECs. For $\mathrm{Sb}_{2} \mathrm{Te}_{3}, I_{i}(T)$ for large- $T$ limit coincides fortuitously for $x y$ biaxial and $z$ uniaxial strains. We also show the values of the macroscopic Grüneisen parameters, $\gamma_{m, i}(T)$ in Fig. 3(b) and (e) (dashed lines). $\mathrm{Bi}_{2} \mathrm{Se}_{3}$ has a large- $T$ limit of $\gamma_{m}$ of 1.27 and 1.09 for the $x y$ biaxial and $z$ uniaxial strains. For $\mathrm{Sb}_{2} \mathrm{Te}_{3}$, the large- $T$ limit of $\gamma_{m}$ is 1.36 for both $x y$ biaxial and $z$ uniaxial strains, which is in good agreement with a reported ${ }^{28}$ result of 1.40. Therefore it is concluded that $\mathrm{Sb}_{2} \mathrm{Te}_{3}$ has a higher phonon anharmonicity than $\mathrm{Bi}_{2} \mathrm{Se}_{3}$ based on the macroscopic Grüneisen parameters.

The linear TECs for $\mathrm{Bi}_{2} \mathrm{Se}_{3}$ in the $a$ and $c$ directions are shown in Fig. 3(c). We observe very good agreement between theory and experiment for $\alpha_{c}$ up to $60 \mathrm{~K}$, beyond which the experiment data shows a dip between 60 and $100 \mathrm{~K}$ and raises slowly after $100 \mathrm{~K}$. The theoretical values for $\alpha_{a}$ are underestimated below $60 \mathrm{~K}$ but a good agreement with experiment is observed between 60 and $180 \mathrm{~K}$. The linear TECs for $\mathrm{Sb}_{2} \mathrm{Te}_{3}$ in Fig. $3(\mathrm{f})$ show a reasonable agreement between theory and experiment for both $\alpha_{a}$ and $\alpha_{c}$ for temperature below $80 \mathrm{~K}$. Finally we note that the theoretical large- $T$ limit of $\alpha_{a}$ for $\mathrm{Bi}_{2} \mathrm{Se}_{3}$ and $\mathrm{Sb}_{2} \mathrm{Te}_{3}$ are the fortuitously the same $\left(11.3 \times 10^{-6} \mathrm{~K}^{-1}\right)$. The large- $T$ limit of $\alpha_{c}$ for $\mathrm{Bi}_{2} \mathrm{Se}_{3}\left(17.4 \times 10^{-6} \mathrm{~K}^{-1}\right)$ and $\mathrm{Sb}_{2} \mathrm{Te}_{3}\left(17.3 \times 10^{-6} \mathrm{~K}^{-1}\right)$ are also very similar. Since the elastic constants for both materials are rather similar, from Eq. 1 we reason that the slightly larger anharmonicity found in $\mathrm{Sb}_{2} \mathrm{Te}_{3}$ is somewhat compensated by its slightly larger primitive cell volume, which results in very similar temperature dependence of linear TECs for $\mathrm{Bi}_{2} \mathrm{Se}_{3}$ and $\mathrm{Sb}_{2} \mathrm{Te}_{3}$. Finally, we note that the complicated temperature dependence of linear TECs in experiments was argued to be attributed to higher-order anharmonic effects and the breaking of the van der Waals bonds between two Se-Se (or Te-Te) layers ${ }^{5}$ at elevated temperatures. We expect the use of quasi-harmonic approximation (QHA) may improve the prediction of the Grüneisen formalism at higher temperatures, however, we do not have enough computational resources for a full QHA treatment for both crystals.
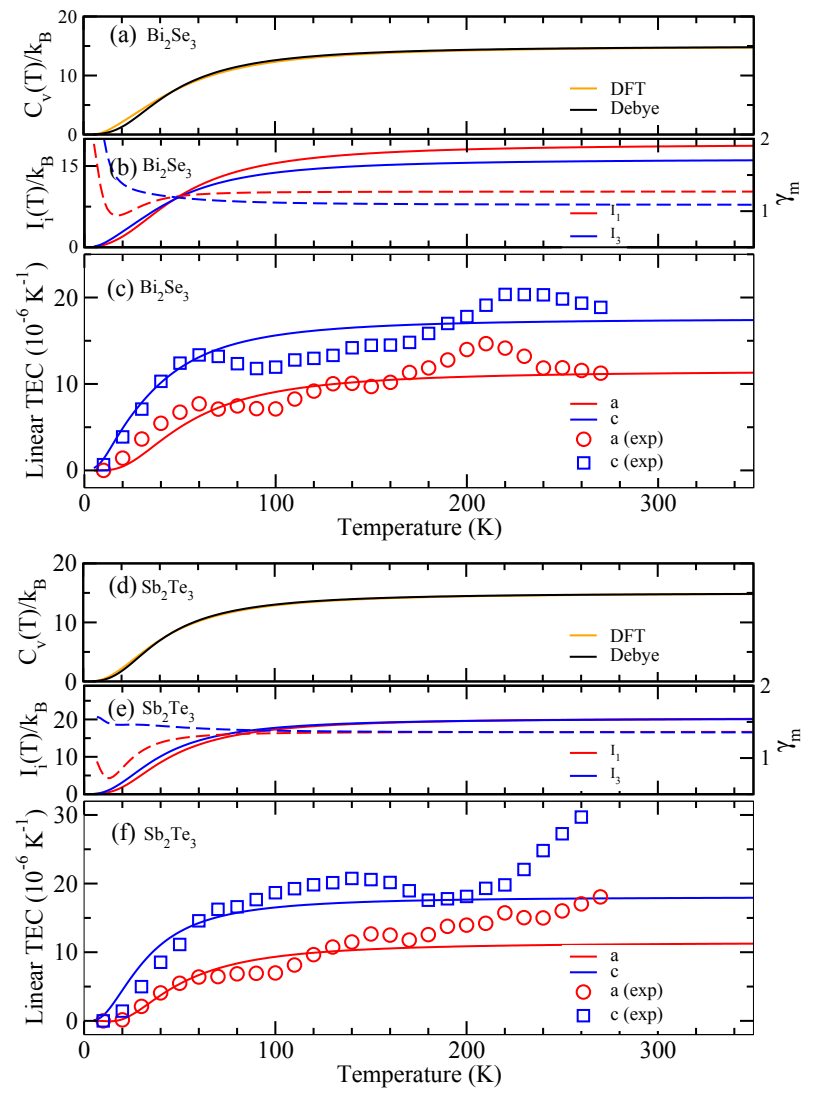

FIG. 3. The temperature dependence of (a) $C_{v}$, (b) $I_{i}$ (solid lines) and $\gamma_{m, i}$ (dashed lines), and (c) the linear TECs of $\mathrm{Bi}_{2} \mathrm{Se}_{3}$. The respective data shown in (d), (e), and (f) are for $\mathrm{Sb}_{2} \mathrm{Te}_{3}$.

\section{SUMMARY}

In summary, we have performed density-functional theory (DFT) calculations to study the phonon anharmonicity of two trigonal systems $\mathrm{Bi}_{2} \mathrm{Se}_{3}$ and $\mathrm{Sb}_{2} \mathrm{Te}_{3}$. Building upon previous computational approaches, we devised an efficient Grüneisen approach in calculating the linear thermal expansion coefficients (TECs). The symmetry of the crystals are fully utilized to reduce the comparatively expensive phonon calculations (compared to standard DFT total-energy calculations) to a minimal set. Even though the main aim of the paper is to study the linear TECs of the systems, many intermediate quantities such as density of phonon states, heat capacity, Debye temperature, mode-dependent Grüneisen parameter, density of Grüneisen parameters, density of phonon states weighted by Grüneisen parameter, and macroscopic Grüneisen parameter have been carefully analyzed to shed light on the temperature dependence of linear TECs of $\mathrm{Bi}_{2} \mathrm{Se}_{3}$ and $\mathrm{Sb}_{2} \mathrm{Te}_{3}$. Reasonably good agreement between theory and experiment for linear TECs has been demonstrated. With the demonstrated accuracy and efficiency of the method, we are confident that a wide applicability of our approach to other thermoelectrics or even other 
classes of low-symmetry materials. We hope our results will encourage the inclusion of our method in accelerated materials search packages.

The raw/processed data required to reproduce these findings cannot be shared at this time as the data also forms part of an ongoing study.

\section{ACKNOWLEDGMENTS}

We gratefully thank the National Supercomputing Center (NSCC), Singapore and A*STAR Computational Resource Center (ACRC), Singapore for computing resources.

${ }^{1}$ G. J. Snyder and E. S. Toberer, Nature Mater. 7, 105 (2008).

${ }^{2}$ M. Z. Hasan and C. L. Kane, Rev. Mod. Phys. 82, 3045 (2010).

${ }^{3}$ H. Zhang, C.-X. Liu, X.-L. Qi, X. Dai, Z. Fang, and S.-C. Zhang, Nature Phys. 5, 438 (2009).

${ }^{4}$ W. Zhang, R. Yu, H.-J. Zhang, X. Dai, and Z. Fang, Nature Phys. 12, 065013 (2010).

${ }^{5}$ X. Chen, H. D. Zhou, A. Kiswandhi, Y. P. Chen, P. A. Sharma, A. L. L. Sharma, M. A. Hekmaty, D. Smirnov, and Z. Jiang, Appl. Phys. Lett. 99, 261912 (2011).

${ }^{6}$ P. Dutta, D. Bhoi, A. Midya, N. Khan, P. Mandal, S. S. Samatham, and V. Ganesan, Appl. Phys. Lett. 100, 251912 (2012).

${ }^{7}$ Y. Tian, S. Jia, R. J. Cava, R. Zhong, J. Schneeloch, G. Gu, and K. S. Burch, Phys. Rev. B 95, 094104 (2017).

${ }^{8}$ D. Das, S. Das, P. Singha, K. Malik, A. K. Deb, A. Bhattacharyya, V. A. Kulbachinski, R. Basu, S. Dhara, S. Bandyopadhyay, and A. Banerjee, Phys. Rev. B 96, 064116 (2017).

${ }^{9}$ G. C. Sosso, S. Caravati, and M. Bernasconi, J. Phys.: Condens. Matter 21, 095410 (2009).
${ }^{10}$ D. Bessas, I. Sergueev, H.-C. Wille, J. Perbon, D. Ebling, and R. P. Hermann, Phys. Rev. B 86, 224301 (2012).

${ }^{11}$ J. Lin, L. Guo, Q. Huang, Y. Jia, K. Li, X. Lai, and X. Chen, Phys. Rev. B 83, 125430 (2011).

${ }^{12}$ Y. Kim, X. Chen, J. Shi, I. Miotkowski, Y. P. Chen, P. A. Sharma, A. L. L. Sharma, M. A. Hekmaty, Z. Jiang, and D. Smirnov, Appl. Phys. Lett. 100, 071907 (2012).

${ }^{13}$ P. K. Schelling and P. Keblinski, Phys. Rev. B 68, 035425 (2003). ${ }^{14}$ N. Mounet and N. Marzari, Phys. Rev. B 71, 205214 (2005).

${ }^{15}$ C. K. Gan, J. R. Soh, and Y. Liu, Phys. Rev. B 92, 235202 (2015).

${ }^{16}$ C. K. Gan and Y. Y. F. Liu, Phys. Rev. B 94, 134303 (2016).

${ }^{17}$ P. Giannozzi, S. Baroni, N. Bonini, M. Calandra, R. Car, C. Cavazzoni, D. Ceresoli, G. L. Chiarotti, M. Cococcioni, I. Dabo, A. Dal Corso, S. de Gironcoli, S. Fabris, G. Fratesi, R. Gebauer, U. Gerstmann, C. Gougoussis, A. Kokalj, M. Lazzeri, L. Martin-Samos, N. Marzari, F. Mauri, R. Mazzarello, S. Paolini, A. Pasquarello, L. Paulatto, C. Sbraccia, S. Scandolo, G. Sclauzero, A. P. Seitsonen, A. Smogunov, P. Umari, and R. M. Wentzcovitch, J. Phys.: Condens. Matter 21, 395502 (2009).

${ }^{18}$ A. M. Rappe, K. M. Rabe, E. Kaxiras, and J. D. Joannopoulos, Phys. Rev. B 41, 1227 (1990).

${ }^{19}$ S. Nakajima, J. Phys. Chem. Solids 24, 479 (1963).

${ }^{20}$ P. Pavone, K. Karch, O. Schütt, W. Windl, D. Strauch, P. Giannozzi, and S. Baroni, Phys. Rev. B 48, 3156 (1993).

${ }^{21}$ C. H. Lee and C. K. Gan, Phys. Rev. B 96, 035105 (2017).

${ }^{22}$ A. Dal Corso, J. Phys.: Condens. Matter 28, 075401 (2016).

${ }^{23}$ W. Setyawan and S. Curtarolo, Comput. Mater. Sci. 49, 299 (2010).

${ }^{24}$ N. W. Ashcroft and N. D. Mermin, Solid State Physics (Saunders College Publishing, New York, 1976).

${ }^{25}$ Y. Liu, K. T. E. Chua, T. C. Sum, and C. K. Gan, Phys. Chem. Chem. Phys. 16, 345 (2014).

${ }^{26}$ T. Tohei, A. Kuwabara, F. Oba, and I. Tanaka, Phys. Rev. B 73, 064304 (2006).

${ }^{27}$ O. Madelung, Semiconductors: Data Handbook (Springer, Berlin, 2004).

${ }^{28}$ R. P. Stoffel, V. L. Deringer, R. E. Simon, R. P. Hermann, and R. Dronskowski, J. Phys.: Condens. Matter 27, 085402 (2015). 